\title{
Enhancing the Coherence of a Spin Qubit by Operating it as a Feedback Loop Controlling its Nuclear Spin Bath
}

\author{
Hendrik Bluhm, , * Sandra Foletti, ${ }^{1}$, 田 Diana Mahalu, ${ }^{2}$ Vladimir Umansky, ${ }^{2}$ and Amir Yacoby ${ }^{1,0}$ \\ ${ }^{1}$ Department of Physics, Harvard University, Cambridge, MA 02138, USA \\ ${ }^{2}$ Braun Center for Submicron Research, Department of Condensed Matter Physics, \\ Weizmann Institute of Science, Rehovot 76100, Israel
}

(Dated: 11/27/10)

\begin{abstract}
In many realizations of electron spin qubits the dominant source of decoherence is the fluctuating nuclear spin bath of the host material. The slowness of this bath lends itself to a promising mitigation strategy where the nuclear spin bath is prepared in a narrowed state with suppressed fluctuations. Here, this approach is realized for a two-electron spin qubit in a GaAs double quantum dot and a nearly ten-fold increase in the inhomogeneous dephasing time $T_{2}^{*}$ is demonstrated. Between subsequent measurements, the bath is prepared by using the qubit as a feedback loop that first measures its nuclear environment by coherent precession, and then polarizes it depending on the final state. This procedure results in a stable fixed point at a nonzero polarization gradient between the two dots, which enables fast universal qubit control.

PACS numbers: 73.21.La, 03.67.Lx, 76.70.Fz
\end{abstract}

Spins in semiconductors are attractive qubits because of their long coherence times [1 3], their electrical control and readout [4], and their potential for scalability [5]. Few-electron quantum dot devices have been used successfully in recent years to demonstrate universal control of electron spin qubits as well as single shot readout [69]. However, interaction of the qubit spin(s) with nearby nuclear spins is a significant source of decoherence in several systems [2 4, 10, 11]. It is therefore very attractive to prepare the spin environment of the electron in a way that mitigates this decoherence. One approach would be to polarize it 12], but the nearly complete polarization required for improved coherence 13 is difficult to achieve.

Here, we present a method to narrow the distribution of the fluctuating nuclear hyperfine field while maintaining a weak polarization. In addition to the reduced decoherence, such a narrowed state is of interest for studying the long-time quantum dynamics arising from the spinbath interaction [14]. Our method relies on first letting the qubit evolve under the influence of the bath. The resulting final state of the qubit controls the effectiveness of a subsequent dynamic nuclear polarization step. The qubit thus acts as a complete feedback loop, and the outcome of its measurement of the controlled variable does not need to be known to the outside world.

Electron-nuclear feedback mechanisms were previously observed in resonance locking experiments under microwave [15] and optical irradiation [16 18]. In Refs. 15, 17], a narrowing of the hyperfine field was inferred from the observed bidirectional polarization keeping the system on resonance, but not experimentally verified. In Ref. [16], narrowing was detected spectroscopically, and Ref. [18] studied an ensemble of optically controlled quantum dots. Here, we directly measure the narrowed distribution of the hyperfine field and the dephasing time, $T_{2}^{*}$, of a single, electrically controlled qubit. $T_{2}^{*}$ is en- hanced by nearly an order of magnitude. In contrast to previous experiments, where the feedback mechanism is intrinsic to the polarization dynamics [19, 20], we have intentionally designed it by manipulating the qubit.

The spin qubit studied in this work employs the $m=0$ subspace of two electron spins in a double quantum dot. The energy splitting between the two basis states depends on the hyperfine field gradient between the two dots. Ref. 21] reported a complete elimination of this gradient and an associated enhancement of $T_{2}^{*}$ in similar devices. However, there is now a more likely interpretation of that experiment in terms of a loss of readout contrast due to a large hyperfine field gradient that accelerates inelastic decay [22]. Furthermore, maintaining a nonzero average field gradient as done here is essential for universal fast electrical control of the qubit [9]

The double quantum dot forming our qubit is created by locally depleting a $90 \mathrm{~nm}$ deep two dimensional electron gas (2DEG) with electrostatic gates [Fig. 1(a)]. Each dot is tunnel coupled to a lead and the inter-dot tunnel coupling is $t_{c} \sim 20 \mu \mathrm{eV}$. The phase space of our qubit is spanned by the singlet $|S\rangle \equiv(|\uparrow \downarrow\rangle-|\downarrow \uparrow\rangle) / \sqrt{2}$ and the triplet $\left|T_{0}\right\rangle \equiv(|\uparrow \downarrow\rangle+|\downarrow \uparrow\rangle) / \sqrt{2}$. The remaining two states, $\left|T_{+}\right\rangle \equiv|\uparrow \uparrow\rangle$ and $\left|T_{-}\right\rangle \equiv|\downarrow \downarrow\rangle$, are split off by the Zeeman energy $E_{Z}=g^{*} \mu_{B} B_{\text {ext }}$ induced by an external magnetic field $B_{\text {ext }}$. Throughout this work, $B_{\text {ext }}=0.7 \mathrm{~T}$ was applied along the $z$-axis, parallel to the 2DEG. The encoding of the qubit in two spins enables fast electrical control via the energy difference, $\varepsilon$, between states with both electrons in one dot and one electron in each dot, respectively [4, 9, 23]. The energies of the four spin states depend on $\varepsilon$ as shown in Fig. 11(c). High frequency coaxial lines connected to two gates GL and GR allow rapid changes of $\varepsilon$.

At $\varepsilon \ll 0$, the electrons are fully separated and acquire a phase at a rate proportional to the magnetic 


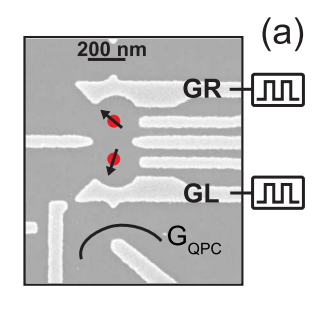

(b)
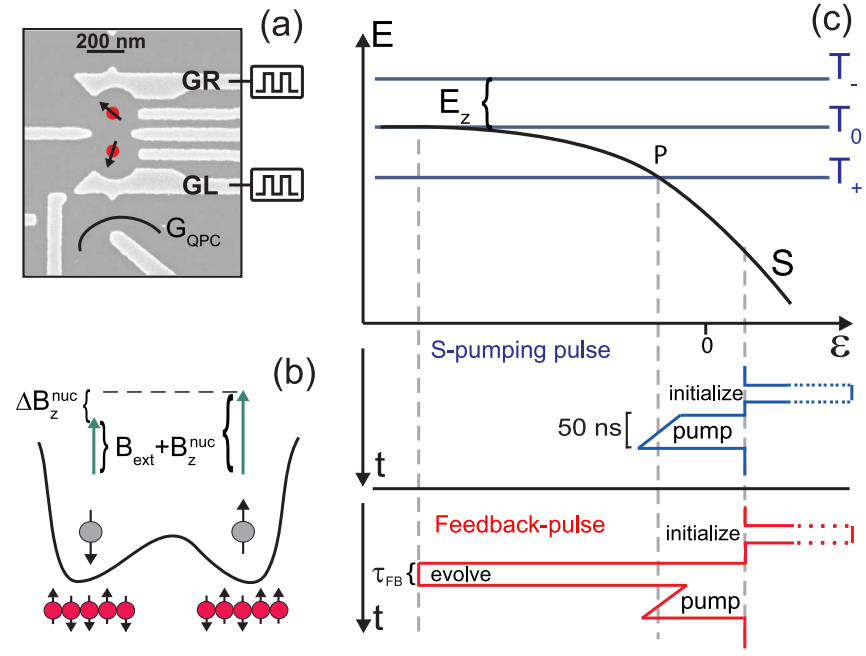

FIG. 1: (color online) Basic principles of the experiment. (a) Scanning electron micrograph of a similar device. The qubit is read out by measuring the conductance $G_{Q P C}$ of the quantum point contact. (b) Illustration of the dynamics for fully separated electrons. The energy splitting between $|\uparrow \downarrow\rangle$ and $|\downarrow \uparrow\rangle$ is proportional to the hyperfine field gradient across the two dots. This level splitting results in coherent oscillations between $|S\rangle$ and $\left|T_{0}\right\rangle$. (c) Top: Energies of the relevant spin states as a function of the detuning $\varepsilon$, which can be controlled with nanosecond time resolution by applying voltage pulses to gates GL and GR. Bottom: Schematic of $\varepsilon(t)$ for the $S$ and feedback pump pulses. The total duration of a single pulse is $250 \mathrm{~ns}$.

field in each dot [Fig. 1(b)]. These local fields are the sum of the homogeneous external field and a hyperfine field proportional to the nuclear polarization parallel to $\mathbf{B}_{e x t}$. Any difference $\Delta B_{n u c}^{z}$ between the two hyperfine fields leads to coherent oscillations of the qubit's state between $|S\rangle$ and $\left|T_{0}\right\rangle$. We probe the hyperfine field gradient, $\Delta B_{n u c}^{z}$, by measuring this free precession using a standard prepare-evolve-measure cycle [4, 8, 9] relying on spin to charge conversion and a quantum point contact for readout. The probability $P_{S}$ of finding the electron in $|S\rangle$ varies sinusoidally with the evolution time $\tau_{S}$ [inset to Fig. 2(a)], with a frequency given by $f=\left|g^{*} \mu_{B} \Delta B_{n u c}^{z}\right| / h$, where $g^{*} \approx-0.4$ is the $g$-factor for a confined electron in GaAs. Fitting a sine curve every time a $\tau_{S}$ sweep is completed yields a time trace of $\left|\Delta B_{n u c}^{z}\right|$ with a sampling rate of about $1 \mathrm{~Hz}[24]$, which is fast enough to probe slow random variations of the nuclear polarization [25].

This near real-time measurement of $\Delta B_{n u c}^{z}$ allows us to use pump cycles discussed in detail in Refs. [9, 21, 26] to compensate the fluctuations of the spin bath. These pump cycles use the degeneracy point of $|S\rangle$ and $\left|T_{+}\right\rangle$ [point P in Fig. 1(c)] to exchange spin between the electrons and the nuclei. In the so called $S\left(T_{+}\right)$pumping cycle, we prepare a $|S\rangle\left(\left|T_{+}\right\rangle\right)$and then sweep trough the $S-T_{+}$transition, which builds up a polarization of the same (opposite) sign as the applied field in each of the dots. How these pump cycles affect $\Delta B_{n u c}^{z}$ depends on the imbalance of the polarization rates in the two dots, which may, for example, arise from different dot sizes 27] due to disorder. Experimentally, we can probe their effect by running on the order of $10^{6}$ cycles (at a $4 \mathrm{MHz}$ repetition rate) between measurements of $\Delta B_{n u c}^{z}$. This alternation between measuring and pumping was applied throughout the remainder of the paper. We find that the two pump cycles always change $\Delta B_{n u c}^{z}$ in opposite directions. If the gradient reaches zero while pumping, it immediately increases again, which suggests a sign change. This behavior is consistent with Ref. [9], where the $T_{+^{-}}$ cycle was first introduced.

To quantify the effect of pumping, we switch between $S$ and $T_{+}$-pumping whenever $\Delta B_{n u c}^{z}$ reaches one of two predetermined limits. This leads to a saw-tooth like time dependence of $\Delta B_{n u c}^{z}$, as shown in Fig. 2(b). Averaging over many such cycles 24] yields the mean rate of change of the gradient, $\Delta B_{n u c}^{z} / d t$, as a function of its value $\Delta B_{n u c}^{z}$ [Fig. 2(d)]. The approximately linear relation for $S$ and $T_{+}$-pumping reflects the relaxation of the polarization due to spin diffusion. For a fixed pump time and pulse, $\Delta B_{n u c}^{z}$ saturates once pumping and relaxation balance each other, but continues to fluctuate on time scales of up to minutes [Fig. 2(a)], with an rmsamplitude $\delta \Delta B_{n u c}^{z}$ of about $3 \mathrm{mT}$ [Fig. [3(c)]. These fluctuations lead to a Gaussian decay of coherent $S-T_{0}$ oscillations after a time $T_{2}^{*}=\hbar \sqrt{2} /\left(g^{*} \mu_{B} \delta \Delta B_{n u c}^{z}\right)=14$ ns when averaging over many $\tau_{S}$ sweeps with different oscillation frequencies [Fig. 3(a)].

Pumping with the $S$ and $T_{+}$-pulse for a fixed time generally does not change $\delta \Delta B_{n u c}^{z}$ appreciably. However, our ability to rapidly measure and manipulate $\Delta B_{n u c}^{z}$ enables us to narrow its distribution using software based feedback. A proportional-integral feedback loop determines the type and duration of pumping between measurements from the time trace of $\Delta B_{n u c}^{z}$. This procedure reduced $\delta \Delta B_{n u c}^{z}$ by about a factor of 2 , corresponding to $T_{2}^{*} \approx 30 \mathrm{~ns}$. It was limited by the $\approx 1 \mathrm{~Hz}$ sampling rate of $\Delta B_{n u c}^{z}$ and could thus be improved with a faster readout technique [8, 28].

While this software feedback method already uses the same qubit to measure and polarize the nuclei, these two tasks are linked via a relatively slow readout process and the measurement computer. In order to speed up the feedback response, we have bypassed this connection by combining both operations into a single pulse derived from the $S$-cycle [Fig. 1(c) bottom]. In such a pulse feedback cycle, the qubit first probes its nuclear environment and then polarizes it depending on the result: after initialization in $|S\rangle$, the qubit is allowed to evolve at $\varepsilon \ll 0$ for a time $\tau_{F B}$. As in the probe cycle, its state oscillates between $|S\rangle$ and $\left|T_{0}\right\rangle$, and the probability of ending in $|S\rangle$ is given by $\left(1+\cos \left(g^{*} \mu_{B} \Delta B_{n u c}^{z} \tau_{F B} / \hbar\right)\right) / 2$. Upon sweeping $\varepsilon$ past the $S-T_{+}$transition, a nuclear spin can only be 


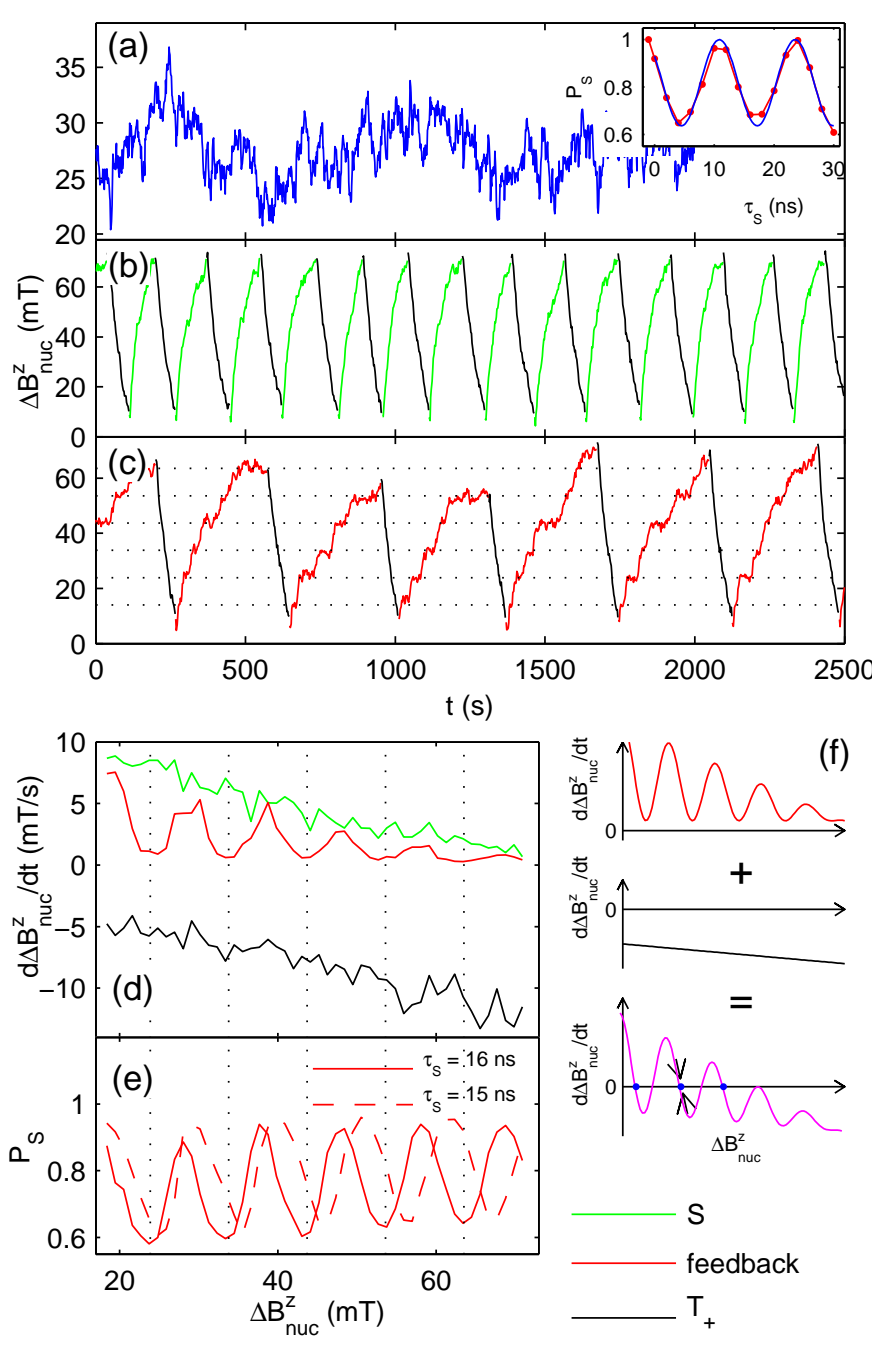

FIG. 2: (color online) Polarization rates. (a) Time trace of $\Delta B_{n u c}^{z}$ showing the fluctuations of the nuclear hyperfine field. The nonzero mean of $\Delta B_{n u c}^{z}$ is due to pumping (without feedback) between measurements. Each data point reflects a fit to the $S-T_{0}$ precession rate as shown in the inset. (b) Same, but alternating between $0.4 \mathrm{~s} S$ and $T_{+}$-pumping between measurements. The legend at the lower right is valid for panels (b)-(f). (c) Same for a feedback pulse with $\tau_{F B}=15 \mathrm{~ns}$ and $T_{+}$. (d) Pump rates extracted by averaging over time traces such as those in panels (b) and (c), but much longer. $d \Delta B_{n u c}^{z} / d t$ is normalized by the time spent pumping. (e) Singlet probability $P_{S}$ after a fixed mixing time $\tau_{S}$ as a function of $\Delta B_{n u c}^{z}$, extracted from the same data as the red curves in panels (c) and (d) [24]. $P_{S}$ is normalized by the change of the charge signal when sweeping gate voltages across the charge transition [9]. It does not decay all the way to zero because of inelastic decay of the metastable $T_{0}$ during the measurement process. The dotted lines in panels (c)-(e) indicate where the polarization stagnates because the qubit is swept through the $S-T_{+}$transition in $\left|T_{0}\right\rangle$. The best match between $P_{S}$ and $d \Delta B_{n u c}^{z} / d t$ is obtained for $\tau_{S}=16 \mathrm{~ns}$, whereas $\tau_{F B}=15 \mathrm{~ns}$. This difference probably reflects finite pulse rise times. (f) Schematic illustration of the summing of the pump rates as shown in panel (d) from $S$ (top) and $T_{+}$-pumping (middle) to obtain stable fixed points (blue dots, bottom). Fluctuations of $\Delta B_{n u c}^{z}$ away from these points are compensated by a restoring pump effect (arrows). flipped by the $|S\rangle$-component of the qubit's state emerging from the evolution. Thus, the pump rate should be proportional to the $\Delta B_{n u c}^{z}$-dependent singlet probability when averaged over many cycles. We have verified this behavior by characterizing the feedback-pulse in the same way as the $S$ and $T_{+}$-pulses. The measured mean pump rate, $d \Delta B_{n u c}^{z} / d t$, oscillates as a function of $\Delta B_{n u c}^{z}$ between 0 and the value corresponding to $S$-pumping [Fig. 2(d)]. As expected, this modulation follows the singlet probability, $P_{S}$, extracted from the same data [Fig. 2(e)]. Its period is given by $h / g^{*} \mu_{B} \tau_{F B}$.

In order to obtain a stable fixed point for $\Delta B_{n u c}^{z}$, the pump rate has to cross zero with a negative slope. Fluctuations of $\Delta B_{n u c}^{z}$ away from the fixed point are then corrected by an opposing pump effect [Fig. 2)(f) bottom]. However, the feedback cycle alone pumps nuclei in one direction only [Fig. 2(d),(f) top]. At the minima of $P_{S}, d \Delta B_{n u c}^{z} / d t$ approaches zero because the qubit is swept past the $S-T_{+}$transition in a $\left|T_{0}\right\rangle$. This stagnation of the polarization results in steplike structures in the time traces in Fig. 2(c). On either side of these points, $d \Delta B_{n u c}^{z} / d t$ remains positive. Thus, $\Delta B_{n u c}^{z}$ continues to grow once a fluctuation pushes it past one of these unstable fixed points [24]. The required sign change can be engineered by preceding the feedback pulse with some amount of $T_{+}$-pumping [24]. The resulting mean pump rate is the sum of a weakly $\Delta B_{n u c}^{z}$-dependent negative $T_{+}$-pulse component and a positive contribution oscillating with $\Delta B_{n u c}^{z}$ from the feedback pulse [ Fig. 2(f)].

To test the stabilizing effect of this pulse combination, we applied it for a fixed time between measurements of $P_{S}$ for different $\tau_{S}$. Figs. [3(b),(d) demonstrate an enhancement of $T_{2}^{*}$ from $14 \mathrm{~ns}$ to $94 \mathrm{~ns}$, and the corresponding narrowing of the distribution of $\Delta B_{n u c}^{z}$ around a fixed point. Here, the pump pulses were applied for 61 ms per $100 \mathrm{~ms}$ interval. The remaining $39 \mathrm{~ms}$ were spent measuring $P_{S}$ for a single $\tau_{S}$, and the data were averaged over 232 sweeps of $\tau_{S}$. We estimate that the pump rate achieved here limits the feedback response time to $\approx 1 \mathrm{~s}$.

In a quantum processor, the measurements could be replaced by a sequence of gate operations, whose fidelity would be substantially improved by the reduction of fluctuations. For a 1 ns $\pi$-rotation generated by a gradient of $100 \mathrm{mT}$ [9] with rms fluctuations of $\delta \Delta B_{n u c}^{z}=0.5 \mathrm{mT}$ as demonstrated here, the fidelity is $\pi^{2} \delta \Delta B_{n u c}^{z}{ }^{2} / 4 \Delta B_{n u c}^{z}{ }^{2} \lesssim 10^{-4}$ [29]. However, due to the slowness of the nuclear bath, an error of order unity accumulates after only 100 such gates. This limitation could be overcome by making gates insensitive to $\delta \Delta B_{n u c}^{z}$ to first order [30]. A pulse angle error of order $\delta \Delta B_{n u c}^{z}{ }^{2} / \Delta B_{n u c}^{z}{ }^{2} \sim 10^{-4}$ would allow $10^{4}$ operations per error. In either case, the improvement in gate fidelity is at least quadratic in the narrowing ratio. Thus, narrowing procedures are very effective at overcoming the limitations imposed by a fluctuating nuclear spin bath. The flexibility of our approach should allow an adaptation to 

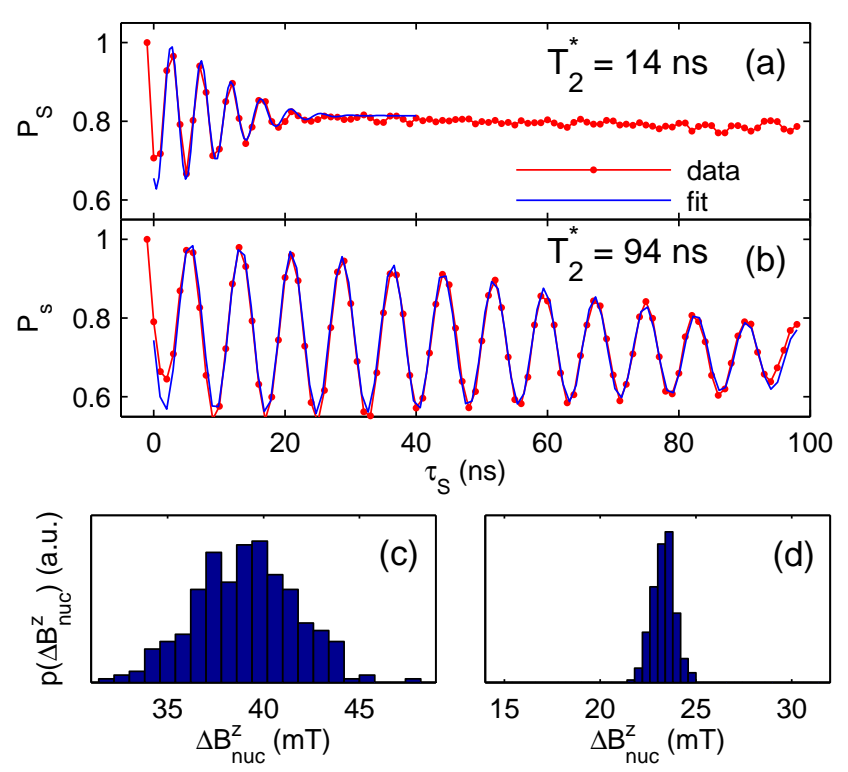

FIG. 3: (color online) $T_{2}^{*}$ enhancement. (a) Ensemble average of $S-T_{0}$ oscillations for fixed pumping $\left(0.7 \mathrm{~s} T^{+}\right.$-pumping and $0.25 \mathrm{~s} S$-pumping repeated every $2.2 \mathrm{~s})$. The pumping maintains a nonzero $\Delta B_{n u c}^{z}=39 \mathrm{mT}$ whose fluctuations lead to a dephasing time of $T_{2}^{*}=14 \mathrm{~ns}$. Other pump times give different oscillation frequencies, but similar dephasing times. $P_{S}$ is normalized as explained in the caption of Fig. 2 (b) Same measurement, but for $30 \mathrm{~ms}$ of feedback pumping with $\tau_{F B}=30 \mathrm{~ns}$ and $31 \mathrm{~ms}$ of $T_{+-}$ pumping after each $39 \mathrm{~ms}$ long measurement. The feedback extends $T_{2}^{*}$ to $94 \mathrm{~ns}$. The blue lines show the fits to $1-P_{S} \propto 1-\cos \left(g^{*} \mu_{B} \Delta B_{n u c}^{z} \tau_{S} / h+\delta\right) e^{-\left(\tau_{S} / T_{2}^{*}\right)^{2}}+\alpha \tau_{S}$ used to extract $T_{2}^{*}$ and $\Delta B_{n u c}^{z}=23 \mathrm{mT}$. The linear term in the fit model accounts for crosstalk of the gate pulses to the charge sensor, and $\delta$ corrects for finite pulse rise times. (c), (d) Corresponding distributions of $\Delta B_{n u c}^{z}$, obtained by histogramming instantaneous values of $\Delta B_{n u c}^{z}$ before ensemble averaging without (c) and with pulse feedback (d).

other systems or other protocols on the same system.

This work was supported by ARO/IARPA, the Department of Defense and the National Science Foundation under Award No. 0653336. This work was performed in part at the Center for Nanoscale Systems (CNS), a member of the National Nanotechnology Infrastructure Network (NNIN), which is supported by the National Science Foundation under NSF Award No. ECS0335765 .

* These authors contributed equally to this work.
† Electronic address: yacoby@physics.harvard.edu

[1] T. D. Ladd, D. Maryenko, Y. Yamamoto, E. Abe, and K. M. Itoh, Phys. Rev. B 71, 014401 (2005).

[2] A. M. Tyryshkin, S. A. Lyon, A. V. Astashkin, and A. M. Raitsimring, Phys. Rev. B 68, 193207 (2003).

[3] L. Childress et al., Science 314, 281 (2006).

[4] J. R. Petta et al., Science 309, 2180 (2005).

[5] J. M. Taylor et al., Nature Physics 1, 177 (2005).

[6] J. Elzerman, R. Hanson, L. H. W. van Beveren, B. Witkamp, L. M. K. Vandersypen, and L. P. Kouwenhoven, Nature 430, 431 (2004).

[7] D. Press, T. Ladd, B. Zhang, and Y. Yamamoto, Nature 456, 218 (2008).

[8] C. Barthel, D. J. Reilly, C. M. Marcus, M. P. Hanson, and A. C. Gossard, Phys. Rev. Lett. 103, 160503 (2009).

[9] S. Foletti, H. Bluhm, D. Mahalu, V. Umansky, and A. Yacoby, Nature Physics 5, 903 (2009).

[10] F. H. L. Koppens et al., Nature 442, 766 (2006).

[11] J. Fischer, W. A. Coish, D. V. Bulaev, and D. Loss, Phys. Rev. B 78, 155329 (2008).

[12] S. Takahashi, R. Hanson, J. van Tol, M. S. Sherwin, and D. D. Awschalom, Phys. Rev. Lett. 101, 047601 (2008).

[13] W. A. Coish and D. Loss, Phys. Rev. B 70, 195340 (2004).

[14] W. A. Coish, J. Fischer, and D. Loss, Phys. Rev. B. 77, 125329 (2008).

[15] I. Vink, K. Nowack, F. Koppens, J. Danon, Y. Nazarov, and L. Vandersypen, Nature Physics 5, 764 (2009).

[16] X. Xu, W. Yao, B. Sun, D. G. Steel, A. S. Bracker, D. Gammon, and L. J. Sham, Nature 459, 1105 (2009).

[17] C. Latta et al., Nature Physics 5, 758 (2009).

[18] A. Greilich et al., Science 317, 1896 (2007).

[19] J. Danon, I. T. Vink, F. H. L. Koppens, K. C. Nowack, L. M. K. Vandersypen, and Y. V. Nazarov, Phys. Rev. Lett. 103, 046601 (2009).

[20] M. S. Rudner and L. S. Levitov, Phys. Rev. Lett. 99, 246602 (2007).

[21] D. J. Reilly, J. M. Taylor, J. Petta, C. M. Marcus, M. P. Hanson, and A. C. Gossard, Science 321, 817 (2008).

[22] C. Barthel, J. Medford, H. Bluhm, A. Yacoby, C. M. Marcus, M. P. Hanson, and A. C. Gossard, in preparation.

[23] J. Levy, Phys. Rev. Lett. 89, 147902 (2002).

[24] See supplementary document in the arXiv ancillary files for additional information on experimental procedures and pump rate measurements.

[25] D. J. Reilly et al., Phys. Rev. Lett. 101, 236803 (2008).

[26] J. R. Petta et al., Phys. Rev. Lett. 100, 067601 (2008).

[27] M. Gullans et al., Phys. Rev. Lett. 104, 226807 (2010).

[28] D. J. Reilly, C. M. Marcus, M. P. Hanson, and A. C. Gossard, App. Phys. Lett. 91, 162101 (2007).

[29] L. Vandersypen and I. Chuang, Rev. Mod. Phys. 76, 1037 (2005).

[30] K. Khodjasteh and L. Viola, Phys. Rev. Lett. 102, 080501 (2009). 